\title{
Nouvelles embauches + annonces de mutations pour thérapeutes ayant un statut d'employé
}

Les nouvelles embauches, les départs et les modifications des données personnelles des thérapeutes ayant un statut d'employé sont à déclarer à l'aide de ce formulaire et à envoyer avec les annexes nécessaires à:

santésuisse, Madame M. Berthel, Römerstrasse 20, 4502 Soleure

\section{Employeur}

Numéro du registre des codes-créanciers:

Nom:

Prénom:

Adresse du cabinet, NPA/lieu:

\section{Emplové}

\section{Nouvelle embauche}

Nom Prénom

Date de naissance Employé depuis le

Pays où a eu lieu la formation ...Année de diplôme

Formation professionnelle: Formation professionnelle: Formation professionnelle: Formation professionnelle: Formation professionnelle: $\square$ Physiothérapeute (y compris copie de la reconnaissance CRS*)
Ergothérapeute (y compris copie de la reconnaissance CRS ${ }^{*}$ )
Diététicien (y compris copie de la reconnaissance CRS*)
Infirmière spécialisée en diabétologie (y compris copie de la reconnaissance ASI**)
Logopédiste (y compris copie du diplôme; pour les étrangers équivalent)
(Veuillez cocher la bonne case)

* CRS Croix rouge suisse

** ASI Association suisse des infirmières et infirmiers

\section{Départs}

No de contrôle:

Nom Prénom

Date de naissance Départ au......

\section{Modifications des données personnelles des collaborateurs actuels}

\section{No de contrôle}

Nom Prénom

Date de naissance Modification au.

PS: Madame M. Berthel est à votre entière disposition pour toute question complémentaire (mardi et vendredi de $8 \mathrm{~h} 30$ à $11 \mathrm{~h} 00$, tél. 03263916 61, fax 0326391678 , e-mail: marion.berthel@santesuisse.ch). 